\title{
Resultados de la tiroidectomía total en el tratamiento del hipertiroidismo
}

\section{Results of total thyroidectomy in the treatment of hyperthyroidism}

\author{
Lourdes Pardo M¹.
}

\begin{abstract}
RESUMEN
Introducción: En el tratamiento del hipertiroidismo se recurre a la cirugía en casos de bocio grande, fracaso del tratamiento médico o indicación social de tratamiento definitivo.

objetivo: Analizar los resultados en cuanto a función tiroidea del tratamiento del hipertiroidismo mediante tiroidectomía total (TT).

Material y método: Estudio retrospectivo de 26 pacientes con hipertiroidismo, intervenidos en el Hospital de la Plana durante 2015-2018, con seguimiento de 36 meses. Tras estudio estadístico descriptivo e inferencial, se analizó la probabilidad de recidiva del hipertiroidismo mediante el método de Kaplan-Meier, y se utilizó el modelo de Cox para ajustar los efectos sobre la recidiva de las diferentes variables.
\end{abstract}

Resultados: El 73,1\% tenían normofunción tiroidea; el 19,2\%, hiperfunción, y en otro $7,7 \%$ existía hipofunción. El tiempo medio de supervivencia (normofunción) fue de 25,87 meses (IC: 17,52-34,21). En el estudio de supervivencia con el log-rank en función de la variable anatomía patológica y complicaciones, no hay diferencias estadísticamente significativas en la supervivencia de ambos grupos.

Discusión: Coincide con otros trabajos publicados.

Conclusión: La TT es un método efectivo de tratamiento para el hipertiroidismo, con baja incidencia de complicaciones. Sin embargo, no hemos encontrado ninguna variable que nos permita predecir el resultado.

Palabras clave: Hipertiroidismo, enfermedad Graves-Basedow, tiroidectomía, normofunción tiroídea, oftalmopatía.

\begin{abstract}
Introduction: Surgery is used in the management of hyperthyroidism in cases of large goiter, failure of medical treatment or social indication of definitive treatment.

Aim: To analyze the results of the treatment of hyperthyroidism by total thyroidectomy (TT) regarding to thyroid function.

Material and methods: Retrospective study of 26 patients with hyperthyroidism, operated on the Hospital de la Plana during 2015-2018, with a follow-up of 36 months. After a descriptive and inferential statistical study, the probability of recurrence of hyperthyroidism was analyzed using the Kaplan-Meier method, and the Cox model was used to adjust the effects on recurrence of the different variables.
\end{abstract}

\footnotetext{
1 Universitat Jaume I, Castellón de la Plana, España.

La autora declara no tener conflictos de interés.

Recibido el 10 de septiembre de 2019. Aceptado el 28 de octubre de 2019.
} 
Results: $73.1 \%$ had thyroid normofunction; in 19.2\% there was hyperfunction, and in another $7.7 \%$ there was hypofunction. The average survival time (normofunction) was 25,87 months (Cl: 17,52-34,21). In the study of survival with the log-rank depending on the pathological results and complications, there are no statistically significant differences in the survival of both groups.

Discussion: This coincides with other published works.

Conclusion: TT is an effective method of treatment for hyperthyroidism, with a low incidence of complications. However, we have not found any variable that allows us to predict the result.

Key words: Hyperthyroidism, Graves-Basedow disease, thyroidectomy, thyroid normofunction, ophthalmopathy

\section{INTRODUCCIÓN}

Numerosos estudios señalan que la causa más frecuente de hipertiroidismo es la enfermedad de Graves-Basedow (EGB), cuyo origen es autoinmune ${ }^{1}$. Otras causas de hipertiroidismo pueden ser los nódulos tiroideos hiperfuncionantes, la tiroiditis, el consumo excesivo de yodo o el consumo excesivo de hormona tiroidea sintética. En el tratamiento del hipertiroidismo se presentan diferentes opciones. De acuerdo con Okosieme², el $\left.\right|^{131}$ es el tratamiento de elección en tiroides pequeños y poco heterogéneos, y en caso de recidivas. El bocio nodular tóxico debe ser tratado con fármacos antitiroideos solo como preparación antes del ${ }^{131}$. Se recurre al tratamiento quirúrgico ${ }^{3}$ en aquellos casos de bocio de gran tamaño, fracaso del tratamiento médico a largo plazo 0 indicación social de tratamiento definitivo, tal como ha publicado Candel ${ }^{4}$. Se incluyen en este último grupo los casos de pacientes con temor a degeneración maligna, motivos estéticos o como alternativa a control médico continuado. Para algunos tipos de hipertiroidismo, tales como un adenoma solitario tóxico, el tratamiento quirúrgico sería una hemitiroidectomía ${ }^{5}$. En los demás casos de hipertiroidismo, incluida la EGB, está indicada la tiroidectomía total.

\section{OBJETIVO}

En este trabajo se pretende analizar los resultados en cuanto a función tiroidea del tratamiento quirúrgico del hipertiroidismo mediante tiroidectomía total.

\section{MATERIAL Y MÉTODO}

Se trata de un estudio retrospectivo de 26 casos de pacientes con hipertiroidismo, intervenidos de tiroidectomía total (TT) en el Servicio de Otorrinolaringología del Hospital de la Plana (Castellón), durante el periodo de 2015 a 2018. El periodo de seguimiento fue de 36 meses. Se han recogido entre otras variables: sexo, edad, antecedentes personales, aspectos clínicos, técnica quirúrgica, tiempos quirúrgicos (TT en un tiempo o conversión a TT tras hemitiroidectomía previa), diagnóstico anátomo-patológico, estancia, bioquímica en el posoperatorio y complicaciones en el mismo. Todos los pacientes firmaron el consentimiento informado previo a la cirugía, que fue digitalizado y adjuntado a la historia clínica electrónica. Así mismo, el estudio se ha realizado preservando los datos confidenciales de los pacientes, y respetando la normativa legal aplicable y las éticas aceptadas internacionalmente, conforme a la Declaración de Helsinki de la AMM y las normas de buena práctica clínica de la Unión Europea.

Los valores de TSH y $T_{4}$ libre $\left(T_{4} L\right)$ que se han considerado correspondían a la última determinación disponible. Tomamos como valores de referencia normal los del laboratorio de nuestro centro (TSH: 0,27-2,5 mUl/l; $\left.T_{4} \mathrm{~L}: 0,7-1,8 \mathrm{ng} / \mathrm{dl}\right)$. Estos datos se han trasladado a variables cuantitativas y cualitativas, y han sido procesados con el programa SPSS versión 25 para Mac. Según los valores de referencia del laboratorio de nuestro centro, hablamos de hipofunción cuando los valores de $T_{4} L$ fueron inferiores a $0,7 \mathrm{ng} / \mathrm{dl}$. Se consideró hipocalcemia cuando los valores de calcio corregido en la mañana siguiente a la intervención fueron inferiores a $8,5 \mathrm{mg} / \mathrm{dl}$.

En primer lugar, se realizó el estudio de normalidad de las variables cuantitativas mediante 
el test de Shapiro-Wilk. A continuación se realizó un análisis descriptivo de la muestra, y un estudio de asociación de las diferentes variables. Para estudiar la relación entre variables cualitativas y cuantitativas, se utilizó el test de Kruskal-Wallis en los casos de variables no paramétricas, así como el Anova de un factor para los casos de variables paramétricas. La asociación entre variables cualitativas se determinó mediante el test de Chi-cuadrado de Spearman, con la corrección de Yates cuando procedía. Seguidamente, se analizó la probabilidad de recidiva del hipertiroidismo mediante el método de Kaplan-Meier, y se utilizó el modelo de Cox para ajustar los efectos sobre la recidiva de las siguientes variables: incidencias del posoperatorio, diagnóstico anátomo-patológico, valor de PTHi posoperatorio, número de tiempos quirúrgicos, hipocalcemia clínica, extensión endotorácica, sexo y oftalmopatía.

\section{RESULTADOS}

De los 26 pacientes estudiados, al finalizar el periodo de seguimiento, el $73,1 \%$ (19 casos) tenían normofunción tiroidea; el 19,2\% (5 casos) presentaban hiperfunción; y en 7,7\% (2 casos) había hipofunción. Con el test de Shapiro-Wilk se comprobó que las variables edad y dosis de levotiroxina, fueron las que seguían una distribución normal, con valores de $p=0,126$ y $p=0,237$. El resto de variables cuantitativas (estancia y tiempo de seguimiento), no seguían una distribución normal. A continuación se realizó un estudio de asociación entre el resultado final y las diferentes variables, que se muestra en la Tabla 1.

La única asociación significativa con la función tiroidea se observó con las variables oftalmopatía y complicaciones (tratándose en todos los casos de parálisis recurrencial). El resto de variables

Tabla 1. Características de los diferentes grupos según resultado

\begin{tabular}{|c|c|c|c|c|c|}
\hline \multicolumn{2}{|l|}{ Variable } & \multirow{2}{*}{$\begin{array}{l}\text { NORMOFUnCIÓN } \\
\text { n (porcentaje) } \\
5(83,3,3 \%) \\
14(70,0 \%)\end{array}$} & \multirow{2}{*}{$\begin{array}{l}\text { HIPERFUNCIÓN } \\
\text { n (porcentaje) } \\
0(0,0 \%) \\
5(25,0 \%)\end{array}$} & \multirow{2}{*}{$\begin{array}{c}\text { HIPOFUNCIÓN } \\
\text { n (porcentaje) } \\
\begin{array}{c}1(16,7 \%) \\
1(5,0 \%)\end{array}\end{array}$} & \multirow{2}{*}{$\begin{array}{c}p \\
0,297\end{array}$} \\
\hline SEXO & $\begin{array}{l}\text { hombre } \\
\text { mujer }\end{array}$ & & & & \\
\hline TSH pos & $\begin{array}{l}\text { disminuido } \\
\text { normal } \\
\text { aumentado }\end{array}$ & $\begin{array}{l}4(80,0 \%) \\
9(75,0 \%) \\
6(66,7 \%)\end{array}$ & $\begin{array}{l}1(20,0 \%) \\
3(25,0 \%) \\
1(11,1 \%)\end{array}$ & $\begin{array}{c}0(0,0 \%) \\
0(0,0 \%) \\
2(22,2 \%)\end{array}$ & 0,356 \\
\hline $\mathrm{T}_{4} \mathrm{~L}$ & $\begin{array}{l}\text { disminuido } \\
\text { aumentado }\end{array}$ & $\begin{array}{c}1(100 \%) \\
18(72,0 \%)\end{array}$ & $\begin{array}{l}0(0,0 \%) \\
5(20 \%)\end{array}$ & $\begin{array}{l}0(0,0 \%) \\
2(8,0 \%)\end{array}$ & 0,826 \\
\hline ENDOTORÁCICO & $\begin{array}{l}\text { no } \\
\text { sí }\end{array}$ & $\begin{array}{l}18(72,0 \%) \\
1(100,0 \%)\end{array}$ & $\begin{array}{c}5(20,0 \%) \\
0(0,0 \%)\end{array}$ & $\begin{array}{l}2(8,0 \%) \\
0(0,0 \%)\end{array}$ & 0,826 \\
\hline TIEMPOS QUIRÚRGICOS & $\begin{array}{l}\text { uno } \\
\text { dos }\end{array}$ & $\begin{array}{l}17(70,8 \%) \\
1(100,0 \%)\end{array}$ & $\begin{array}{c}5(20,8 \%) \\
0(0,0 \%)\end{array}$ & $\begin{array}{l}2(8,3 \%) \\
0(0,0 \%)\end{array}$ & 0,817 \\
\hline PTHi INTRA & $\begin{array}{l}\text { disminuida } \\
\text { normal }\end{array}$ & $\begin{array}{c}7(87,5 \%) \\
12(66,7 \%)\end{array}$ & $\begin{array}{l}1(12,5 \%) \\
4(22,2 \%)\end{array}$ & $\begin{array}{c}0(0,0 \%) \\
2(11,1 \%)\end{array}$ & 0,475 \\
\hline HIPOCALCEMIA SUBCLÍNICA & $\begin{array}{l}\text { no } \\
\text { sí }\end{array}$ & $\begin{array}{c}14(73,7 \%) \\
5(71,4 \%)\end{array}$ & $\begin{array}{l}3(15,8 \%) \\
2(28,6 \%)\end{array}$ & $\begin{array}{c}2(10,5 \%) \\
0(0,0 \%)\end{array}$ & 0,556 \\
\hline HIPOCALCEMIA CLÍNICA & $\begin{array}{l}\text { no } \\
\text { sí }\end{array}$ & $\begin{array}{c}16(72,7 \%) \\
3(75,0 \%)\end{array}$ & $\begin{array}{l}4(18,2 \%) \\
1(0250 \%)\end{array}$ & $\begin{array}{l}2(9,1 \%) \\
0(0,0 \%)\end{array}$ & 0,799 \\
\hline NORMOCALCEMIA & $\begin{array}{l}\text { sí } \\
\text { no }\end{array}$ & $\begin{array}{l}12(70,6 \%) \\
7(77,8 \%)\end{array}$ & $\begin{array}{l}3(17,6 \%) \\
2(22,2 \%)\end{array}$ & $\begin{array}{l}2(11,8 \%) \\
2(0,0 \%)\end{array}$ & 0,559 \\
\hline COMPLICACIONES & $\begin{array}{l}\text { ninguna } \\
\text { parálisis recurrente }\end{array}$ & $\begin{array}{c}12(80,0 \%) \\
7(63,6 \%)\end{array}$ & $\begin{array}{l}3(20,0 \%) \\
2(18,2 \%)\end{array}$ & $\begin{array}{c}0(0,0 \%) \\
2(18,2 \%)\end{array}$ & 0,019 * \\
\hline LUGOL & $\begin{array}{l}\text { no } \\
\text { sí }\end{array}$ & $\begin{array}{c}6(85,7 \%) \\
13(68,4 \%)\end{array}$ & $\begin{array}{l}1(14,3 \%) \\
4(21,1 \%)\end{array}$ & $\begin{array}{c}0(0,0 \%) \\
2(10,5 \%)\end{array}$ & 0,586 \\
\hline ANATOMÍA PATOLOGICA & $\begin{array}{l}\text { benigna } \\
\text { maligna }\end{array}$ & $\begin{array}{c}18(75,0 \%) \\
1(50,0 \%)\end{array}$ & $\begin{array}{l}4(16,7 \%) \\
1(50,0 \%)\end{array}$ & $\begin{array}{l}2(8,3 \%) \\
0(0,0 \%)\end{array}$ & 0,499 \\
\hline OFTALMOPATÍA & $\begin{array}{l}\text { sí } \\
\text { no }\end{array}$ & $\begin{array}{c}0(0,0 \%) \\
19(79,2 \%)\end{array}$ & $\begin{array}{l}2(100 \%) \\
3(12,5 \%)\end{array}$ & $\begin{array}{l}0(0,0 \%) \\
2(8,3 \%)\end{array}$ & $0,011^{*}$ \\
\hline Total & & $19(73,1 \%)$ & $5(19,2 \%)$ & $2(7,7 \%)$ & \\
\hline
\end{tabular}

*Nivel de significación $p<0,05$. 
cuantitativas tampoco mostró asociación significativa.

En la curva de Kaplan-Meier (Figura 1), el tiempo de seguimiento se expresó en meses, siendo su valor de 36. Como corresponde a este tipo de estudios, hay fechas muy bien definidas de inicio y de cierre, pero los sujetos se incorporan al estudio en momentos diferentes.

El comienzo de la fecha de seguimiento fue el día de la intervención quirúrgica y la fecha de finalización fue la de la última visita a endocrinología. Como variable estado, es decir el evento a estudiar, se consideró una alteración de la normofunción, incluyendo los casos de hiperfunción e hipofunción. Del total de pacientes, en 7 estuvo alterado, mientras que el porcentaje de censurados fue de $73,1 \%$ (19 casos). El tiempo medio de supervivencia (que en este caso se traduce como normofunción), fue de 25,87 meses. Corresponde al tiempo en el que el $50 \%$ de los sujetos siguen sin haber desarrollado el evento. En las Figuras 2 y 3 se muestra el estudio de supervivencia en función de la variable anatomía patológica y complicaciones.

Para hacer el log rank, se consideraron diferentes factores, que se expresan en la Tabla 2, siendo solo significativo para las variables anatomía pato-

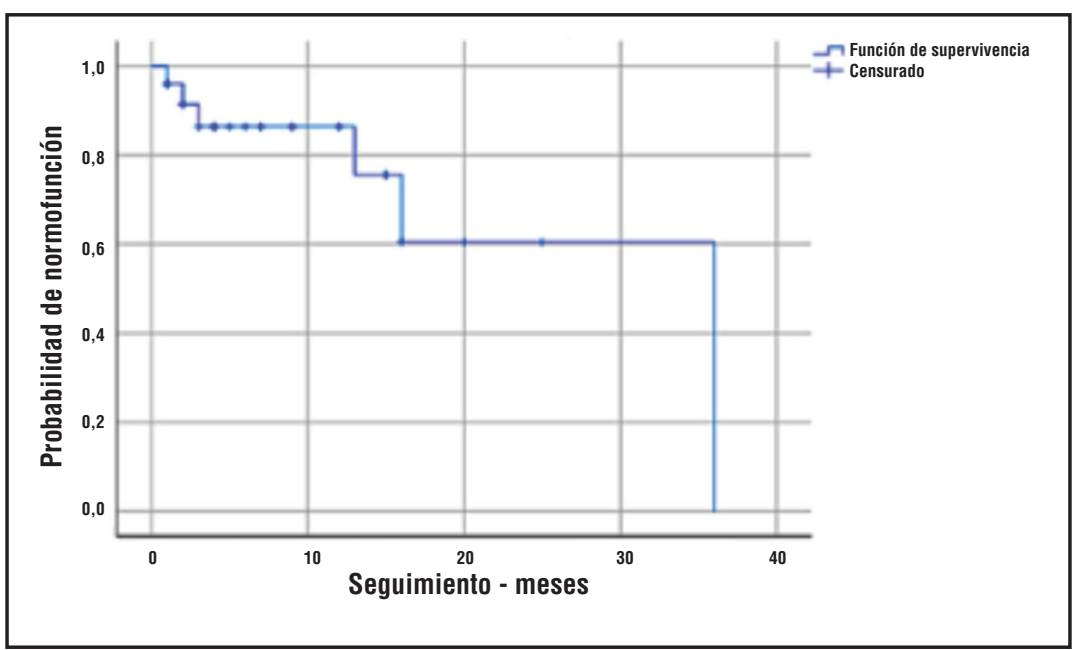

Figura 1. Curva de Kaplan-Meier con función de supervivencia.

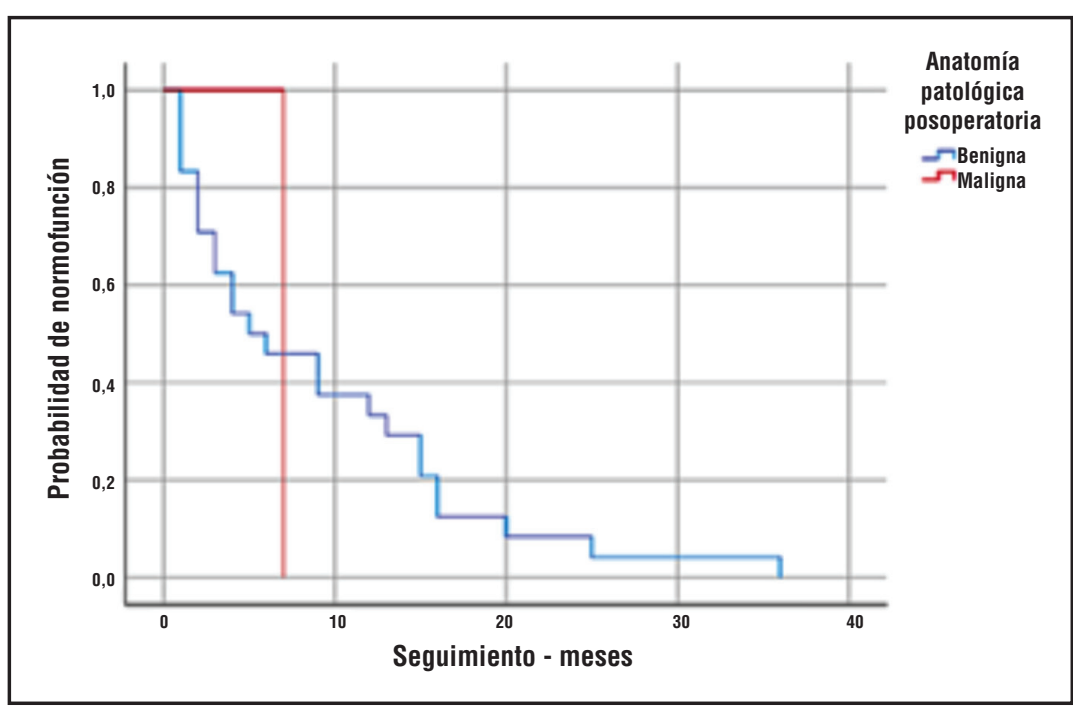

Figura 2. Estudio de supervivencia ajustado según diagnóstico anátomo-patológico. 


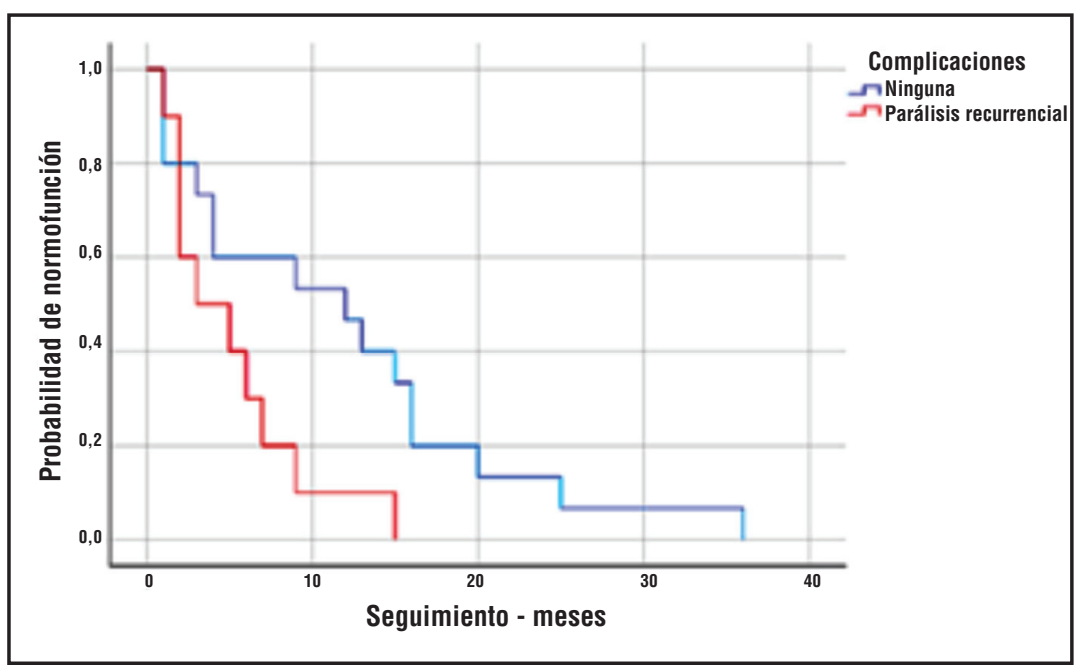

Figura 3. Estudio de supervivencia ajustado según complicaciones en el posoperatorio.

Tabla 2. Log Rank/Mantel-Cox

\begin{tabular}{|ll|}
\hline Variable & $p$ \\
\hline Sexo & 0,373 \\
Hipocalcemia subclínica & 0,879 \\
Hipocalcemia clínica & 0,980 \\
Normocalcemia & 0,478 \\
Anatomía patológica & $0,050^{*}$ \\
Complicaciones & $0,007^{*}$ \\
Preparación quirúrgica & 0,574 \\
Oftalmopatía & 0,105 \\
TSH última & 0,339 \\
T4L última & 0,762 \\
Tiempos quirúrgicos & 0,694 \\
PTHi & 0,696 \\
\hline
\end{tabular}

*Nivel de significación $p<0,05$.

lógica posoperatoria (benigna y maligna) y complicaciones (que en todos los casos correspondió a parálisis recurrencial).

En el análisis multivariable mediante regresión de Cox (Tabla 3), ninguna de las variables analizadas consiguió significación estadística, por lo que ninguna de ellas (complicaciones, diagnóstico anátomo-patológico, valor de PTH posoperatorio, número de tiempos quirúrgicos, hipocalcemia clínica, extensión endotorácica, sexo y oftalmopatía) influye en la supervivencia del evento estudiado. Posteriormente se analizó el comportamiento de los residuales del modelo estimado para verificarlo, comprobando que se cumple el supuesto de proporcionalidad de los riesgos y que no hay valores atípicos.

\section{DISCUSIÓN}

Aunque la etiología del hipertiroidismo puede ser variada, la causa más frecuente es la EGB. Es bien conocido que la EGB es una afección multisistémica, de patogenia autoinmune y caracterizada clínicamente por hiperplasia difusa de la glándula tiroidea con hiperfunción, oftalmopatía infiltrativa y, en ocasiones, mixedema pretibial $^{6}$. Su prevalencia es de aproximadamente 
Tabla 3. Análisis multivariante mediante regresión de Cox

\begin{tabular}{|lcccccc|}
\hline Variable & B & SE & Wald & df & Sig & HR \\
\hline Complicaciones & $-1,123$ & 0,646 & 3,025 & 1 & 0,082 & 0,325 \\
Anatomía patológica & $-0,412$ & 1,294 & 0,102 & 1 & 0,750 & 0,662 \\
PTHi intraoperatoria & 1,483 & 0,743 & 3,988 & 1 & 0,066 & 4,407 \\
Hipocalcemia clínica & 1,513 & 0,855 & 3,130 & 1 & 0,077 & 4,539 \\
Extensión endotorácica & $-0,147$ & 1,293 & 0,013 & 1 & 0,909 & 0,863 \\
Sexo & $-0,506$ & 0,694 & 0,533 & 1 & 0,465 & 0,603 \\
Oftalmopatía & 0,13 & 0,836 & 0,000 & 1 & 0,988 & 1,013 \\
\hline
\end{tabular}

B: valor del coeficiente en el modelo de Cox (Bi); SE: error estándar; Wald: valor del estadístico de Wald; df: grados de libertad; Sig: nivel de significación del estadístico de Wald; HR: hazard ratio.

Descripción de las variables: complicaciones: sí/no; anatomía patológica: benigna/maligna; PTHi intraoperatoria: disminuida/normal; hipocalcemia clínica: sí/no; extensión endotorácica: sí/no; sexo: hombre/mujer.

el $0,5 \%$ de la población. Predomina en la tercera y la cuarta décadas de la vida y afecta sobre todo a la mujer (10:1), siendo las características de nuestra muestra bastante similares, con una relación mujer:hombre de 20:6, y una edad media de 51,5 años (mínimo 30 y máximo 78). La hiperfunción tiroidea depende de la producción de autoanticuerpos estimulantes de la glándula tiroides en una población predominantemente femenina predispuesta a través, en parte, de los genes del sistema HLA. También la oftalmopatía surge por un mecanismo autoinmune dirigido contra la musculatura ocular extrínseca?

En el curso de la enfermedad pueden surgir diversas complicaciones. La cardiopatía tirotóxica, que se presenta más frecuentemente en pacientes con algún tipo de cardiopatía subyacente, puede manifestarse en forma de trastornos del ritmo, insuficiencia cardíaca o insuficiencia coronaria. La exoftalmía maligna constituye una complicación que puede conducir incluso a la ceguera ${ }^{8}$. La crisis tirotóxica es una complicación muy grave, pero por fortuna rara. Ali ${ }^{9}$ publica buenos resultados en la tiroidectomía total practicada con carácter de urgencia en los casos de tirotoxicosis por EGB.

Para el diagnóstico definitivo de la enfermedad, habitualmente es suficiente la determinación de las concentraciones de T4 libre (aumentadas) y de TSH (disminuidas). La determinación de la TSH basal es un método muy útil y sensible, en ocasiones más que la determinación de T4 libre. La infrecuente aparición de tirotoxicosis únicamente por T3 no justifica su determinación sistemática, aunque debe solicitarse si la sospecha de tiro- toxicosis es elevada y la T4 libre es normal ${ }^{10}$. En diversas circunstancias es útil la determinación de anticuerpos antirreceptor de la TSH, si bien no lo hemos recogido en nuestro estudio para evitar sesgos, dado que la muestra es reducida y no todos los pacientes disponían de esta determinación.

La EGB abandonada a su evolución espontánea es un proceso grave, con una importante mortalidad. Sometida a tratamiento adecuado, su pronóstico es favorable, aunque el control de su evolución plantea con frecuencia problemas que a menudo obligan a realizar tratamientos radicales con destrucción de la glándula por medios quirúrgicos o radioisotópicos ${ }^{11}$. Como se ha señalado, en la actualidad se dispone de tres tipos de medidas: la administración de fármacos tirostáticos, la tiroidectomía y el empleo de radioyodo. El tratamiento quirúrgico consiste en la tiroidectomía. Gan ${ }^{12}$ expuso el papel de la cirugía en el tratamiento quirúrgico de la patología autoinmune del tiroides. En nuestro caso, siempre se ha realizado TT. Tal como señala Liư ${ }^{13}$, la TT es más eficaz que la tiroidectomía subtotal para prevenir el hipertiroidismo recurrente en la EGB (8 ó 9 pacientes de 1.000 presentarán recurrencia del hipertiroidismo después de la tiroidectomía total, en comparación con 55 a 67 pacientes de 1.000 después de la tiroidectomía subtotal).

Por último, la administración de ${ }^{131}$ (50-80 $\mathrm{mCi} / \mathrm{g}$ de tejido tiroideo) en dosis fraccionadas puede ofrecer excelentes resultados. Tabuenca ${ }^{14}$ estudió también los factores que influencian el resultado del tratamiento con I ${ }^{131}$ en el hipertiroidismo secundario a bocio nodular tóxico. 
En las formas graves de oftalmopatía infiltrativa se utilizan grandes dosis de glucocorticoides (250-500 mg de metilprednisolona i.v. diluidos en suero fisiológico, en 60-90 min una vez a la semana, durante 6 semanas consecutivas), radioterapia o inmunosupresores $y$, en caso de ineficacia, deberá recurrirse a la descompresión orbitaria. Okolie ${ }^{15}$ describió la inusual asociación de exoftalmos y bocio multinodular. El tipo de cirugía tiroidea realizada no afecta la regresión de la oftalmopatía de Graves ${ }^{13}$. Como indican Alvarez ${ }^{16}$ y cols, se encuentra una incidencia elevada de cáncer tiroideo en pacientes sometidos a tiroidectomía total por hipertiroidismo (22\%). You ${ }^{17}$ expone también la elevada prevalencia y agresividad del carcinoma papilar en pacientes intervenidos por Graves-Basedow (14\%). En nuestro caso, el resultado de la anatomía patológica definitiva fue: 5 casos de hiperplasia nodular (19,23\%); 7 casos de Graves-Basedow $(26,92 \%)$, un adenoma folicular $(3,84 \%)$, un caso de tiroiditis de Hashimoto (3,84\%); y 2 casos de malignidad (un carcinoma papilar y un carcinoma folicular), que corresponde al $7,69 \%$.

La buena respuesta al tratamiento quirúrgico publicada por Marques ${ }^{18}$ se dio en $77 \%$ de casos. En el estudio de Tamatea ${ }^{19}$, se logró normofunción tiroidea solo en el $48 \%$ de los casos tras seguimiento de 1 año, y del $63,5 \%$ a los 10 años de seguimiento. En nuestro estudio se logró la normofunción en 19 casos $(73,07 \%)$, con un periodo de seguimiento de 36 meses. Amat ${ }^{20}$ y cols demostraron que el remanente tiroideo es la única variable que condiciona la función tiroidea posoperatoria. En nuestro trabajo ninguna variable consiguió una significación estadística significativa.

Akram $^{21}$ ha publicado las complicaciones de su casuística: hipocalcemia transitoria $(30,4 \%$ $25,5 \%)$, hipoparatiroidismo prolongado (0,98\%$4,3 \%$ ); disfonía (10,8\%-7,5\%). Nuestro porcentaje de hipocalcemia subclínica fue del 26,92\%, la hipocalcemia clínica apareció en el 15,38\%, presen- tando normocalcemia el 65,38\%. Según Kwon ${ }^{22}$ y otros autores ${ }^{23}$ existe un riesgo más elevado de complicaciones posoperatorias tras TT en los casos de EGB. Si bien Philippe ${ }^{24}$ ha expuesto los criterios que utiliza para realizar la TT como procedimiento ambulatorio, nosotros siempre hemos realizado el procedimiento con ingreso hospitalario, con una estancia media de 1,8 días con un rango entre 1 y 8 días. Fan ${ }^{25}$ expuso los factores de riesgo para la formación de un hematoma cervical tras TT que requirieron revisión quirúrgica. En nuestra casuística no aparece ningún caso de sangrado en el posoperatorio, siendo la principal complicación la disfonía, que apareció en el 34,61\%.

Al Qubaisi ${ }^{26}$ enfatiza la asociación entre hipocalcemia postiroidectomía total y EGB. Entre los pacientes con hipocalcemia, el 16,3\% tenían $\mathrm{EGB}$, mientras que en los pacientes sin EGB, la incidencia de hipocalcemia fue del 9,4\%. Los datos de nuestro trabajo muestran que la hipocalcemia clínica en el posoperatorio inmediato no se asocia con mayor riesgo de hipofunción tiroidea durante el periodo de seguimiento. Hemos empleado lugol en el preoperatorio en 19 casos. No se ha utilizado cuando se trataba de hipertiroidismo subclínico. Tsai $^{27}$ refiere disminución de la vascularización tiroidea y sangrado intraoperatorio con la utilización del mismo, aunque no observa diferencias en cuanto a las complicaciones posoperatorias.

\section{CONCLUSIÓN}

Si bien nuestra serie es reducida, coincidimos con Cipolla ${ }^{28}$ en concluir que la TT es un método efectivo de tratamiento para el hipertiroidismo, que se asocia con una baja incidencia de complicaciones posoperatorias, la mayoría de las cuales son transitorias. En la mayoría de los casos ofrece un control del hipertiroidismo rápido y definitivo. Sin embargo, no hemos encontrado ninguna variable que nos permita predecir el resultado. 


\section{BIBLIOGRAFÍA}

1. Smithson M, Asban A, Miller J, Chen H. Considerations for Thyroidectomy as Treatment for Graves Disease. Clin Med Insights Endocrinol Diabetes 2019; 12. doi: 10.1177/1179551419844523.

2. Okosieme OE, Taylor PN, Evans C, Thayer D, Chal A, Khan I, Draman MS, Tennant B, Geen J, Sayers A, French R, Lazarus JH, Premawardhana LD, Dayan CM. Primary therapy of Graves' disease and cardiovascular morbidity and mortality: a linked-record cohort study. Lancet Diabetes Endocrinol 2019; 7: 278-87.

3. Sugino K, Nagahama M, Kitagawa W, Ohikuwa K, Uruno T, Matsuzu K, Suzuki A, Tomoda C, Y Hames K, AKalshI J, Masaki C, OGIMI Y, Yabuta T, Ito K. Change of surgical strategy for Graves' disease from subtotal thyroidectomy to total thyroidectomy: a single institutional experience. Endocr J 2019; 66: 181-6.

4. Candel MF, Flores B, Soria V, Albarracín A, Miguel J, Andrés B, Illán F, Aguayo Jl. Efectividad y seguridad de la tiroidectomía total en el tratamiento de la enfermedad de GravesBasedow. Cir Esp 2003; 74: 30-2.

5. Ross DS, Burch HB, Cooper DS, Greenlee MC, Laurberg P, Maia AL, Rivkees Sa, Samuels M, Sosa JA, Stan MN, Walter MA. American Thyroid Association Guidelines for Diagnosis and Management of Hyperthyroidism and Other Causes of Thyrotoxicosis. Thyroid 2016; 26 : 1343-421.

6. Alia E, Feit EJ, Levitt JO. Electrosurgical debulking of pretibial myxedema of the foot. Dermatol Online J 2019; 25(2).

7. Sim SY, Lethem C, Coppini DV. Characteristics and outcomes of patients with hyperthyroidism attending a hospital endocrine clinic-A retrospective study. Endocrinol Diabetes Metab 2018;2: e00046. doi: 10.1002/edm2.46.

8. Roos JCP, Murthy R. Update on the clinical assessment and management of thyroid eye disease. Curr Opin Ophthalmol 2019; 30: 401-6.

9. Ali A, Debono M, Balasubramanian SP. Outcomes After Urgent Thyroidectomy Following Rapid Control of Thyrotoxicosis in Graves' Disease are Similar to Those After Elective Surgery in Well-Controlled Disease. World J Surg 2019; 43: 3051-8.
10. Fu H, Cheng L, Jin Y, Chen L. Thyrotoxicosis with concomitant thyroid cancer. Endocr Relat Cancer 2019 (En prensa). doi: 10.1530/ERC-19-0129.

11. Wong KK, Shulkin BL, Gross MD, Avram AM. Efficacy of radioactive iodine treatment of graves' hyperthyroidism using a single calculated 1311 dose. Clin Diabetes Endocrinol 2018; 4: 20. doi: 10.1186/s40842-018-0071-6.

12. Gan T, Randle RW. The Role of Surgery in Autoimmune Conditions of the Thyroid. Surg Clin North Am 2019; 99: 633-48.

13. Liu Z, Masterson L, Fish B, Jani P, Chatterjee K. Thyroid surgery for Graves' disease and Graves' ophthalmopathy. Cochrane Database of Systematic Reviews 2015; 11: CD010576.

14. Tabuenca-Dopico 0, Boente-Varela R, LamasFerReIRo JL. Study of factors that influence the outcome of 131 I treatment in hyperthyroidism secondary to nodular goitre. Rev Esp Med NuCl Imagen Mol 2017; 36: 356-61.

15. Okolie K, Chen D, Ghabrial R, Schmidli R. Exophthalmos and multinodular goitre, an unusual combination. Endocrinol Diabetes Metab Case Rep 2019; 2019. doi: 10.1530/EDM-180138.

16. Alvarez Al, Mulder M, Handelsman RS, Lew Ji, FARRA JC. High Rates of Underlying Thyroid Cancer in Patients Undergoing Thyroidectomy for Hyperthyroidism. J Surg Res 2019; 245: 523-8.

17. You E, Mascarella Ma, Al Jassim A, Forest VI, Hier MP, Tamilia M, Pusztaszeri M, Payne RJ. Prevalence and aggressiveness of papillary thyroid carcinoma in surgically-treated graves' disease patients: a retrospective matched cohort study. Otolaryngol Head Neck Surg 2019; 48: 40. doi: 10.1186/s40463-019-0364-5.

18. Marques 0, Antunes A, Oliveira MJ. Treatment of Graves' disease in children: The Portuguese experience. Endocrinol Diabetes Nutr 2018; 65: 143-9.

19. Tamatea JaU, Tu'akol K, Meyer-Rochow GY, Conaglen JV, Elston MS. Inequitable Long-Term Outcomes for an Indigenous Population After Definitive Treatment of Patients With Graves Disease. J Endocr Soc 2019; 3: 1335-44.

20. Аmat M, Gómez JM, Biondo S, Rafecasa A, JAURRIETA E. Factores pronósticos en el resultado funcional del tratamiento quirúrgico de la 
enfermedad de Graves-Basedow. Med Clínica 2001; 116: 487-90.

21. Akram S, Elfenbein DM, Chen H, Schneider DF, SipPel RS. Assessing American Thyroid Association Guidelines for Total Thyroidectomy in Graves' Disease. J Surg Res 2019; 245: 64-71.

22. Kwon H, KIM JK, LIM W, Moon BI, PaIK NS. Increased risk of postoperative complications after total thyroidectomy with Graves' disease. Head Neck 2019; 41: 281-5.

23. Pardo L, Sánchez P. Factores perioperatorios relacionados con la hipocalcemia postiroidectomía total. Revista Faso 2018; 25: 6-9.

24. Philippe JB, Riou J, Lemoult A, Sarfati-Lebreton M, Hamy A, Muccı S. Feasibility criteria for total thyroidectomy in outpatient surgery. Ann Endocrinol (Paris) 2019; 80: 286-92.

25. Fan C, Zhou X, Su G, Zhou Y, Su J, Luo M, Lı $H$. Risk factors for neck hematoma requiring surgical re-intervention after thyroidectomy: a systematic review and meta-analysis. $B M C$ Surg 2019; 19: 98. doi: 10.1186/s12893-0190559-8.

26. AL QuBaISI M, HaIGH PI. Hypocalcemia after Total Thyroidectomy in Graves Disease. Perm J 2019; 23. doi: 10.7812/TPP/18-188.

27. Tsai CH, Yang PS, Lee JJ, Liu TP, Kuo CY, Cheng SP. Effects of Preoperative lodine Administration on Thyroidectomy for Hyperthyroidism: A Systematic Review and Meta-analysis. Otolaryngol Head Neck Surg 2019; 160: 9931002.

28. Cipolla C, Graceffa G, Calamia S, Fiorentino E, Pantuso G, VienI S, Latteri M. The value of total thyroidectomy as the definitive treatment for Graves' disease: A single centre experience of 594 cases. J Clin Transl Endocrinol 2019; 16: 100183. 